\title{
The EMPOWER-SUSTAIN e-Health Intervention to improve patient activation and self-management behaviours among individuals with Metabolic Syndrome in primary care: study protocol for a pilot randomised controlled trial
}

Maryam Hannah Daud

Universiti Teknologi MARA

Anis Safura Ramli ( $\square$ rossanis_yuzadi@yahoo.co.uk)

Institute of Pathology, Laboratory and Forensic Medicine (I-PPerForM), Universiti Teknologi MARA

(UiTM), Sungai Buloh Campus https://orcid.org/0000-0002-9517-1413

Suraya Abdul-Razak

Universiti Teknologi MARA

Mohamad Rodi Isa

Universiti Teknologi MARA

Fakhrul Hazman Yusoff

Universiti Teknologi MARA

Noorhida Baharudin

Universiti Teknologi MARA

Mohamed Syarif Mohamed-Yassin

Universiti Teknologi MARA

Siti Fatimah Badlishah-Sham

Universiti Teknologi MARA

Azlina Wati Nikmat

Universiti Teknologi MARA

Nursuriati Jamil

Universiti Teknologi MARA

Hapizah Mohd-Nawawi

Universiti Teknologi MARA

Study protocol

Keywords: e-health intervention, self-management, patient activation, chronic care model, chronic disease management, multifaceted intervention, metabolic syndrome, primary care 
Posted Date: February 19th, 2020

DOI: https://doi.org/10.21203/rs.2.17154/v2

License: (c) (i) This work is licensed under a Creative Commons Attribution 4.0 International License. Read Full License

Version of Record: A version of this preprint was published at Trials on April 5th, 2020. See the published version at https://doi.org/10.1186/s13063-020-04237-x. 


\section{Abstract}

Background: Epidemiological studies from various parts of the world have clearly demonstrated that Metabolic Syndrome (MetS) is an increasing global health problem, not only in the western societies but also in the Asian populations. Web-based and mobile phone-based self-management applications have been proven to be effective in improving self-management behaviour in patients with MetS components i.e. diabetes or hypertension. However, evidence is lacking in terms of its effectiveness specifically for patients with MetS. The aim of this pilot study is to evaluate the feasibility and potential effectiveness of the EMPOWER-SUSTAIN @ Self-Management e-Health Intervention in improving patient activation and self-management behaviours among patients with MetS. This paper presents the study protocol.

Methods: A pilot randomised controlled trial will be conducted in a university primary care clinic. A total of 232 patients aged 18-60 years old with MetS will be recruited; 116 will be randomised to receive the EMPOWER-SUSTAIN ( intervention for 6 months and another 116 patients will continue with usual care. The EMPOWER-SUSTAIN ( $\odot$ intervention is a multifaceted chronic disease management strategy based on the Chronic Care Model and persuasive technology theory. It consists of training primary care physicians, nurse and patients to use the EMPOWER-SUSTAIN web-based self-management mobile app (c) , strengthening patient-physician relationship and reinforcing the use of relevant clinical practice guidelines to guide management and prescribing. The primary outcome is the mean change in patient activation score using the Patient Activation Measure ${ }^{\circledR}$ short form Malay version (PAM $®-13-M$ ) questionnaire. The secondary outcomes include the change in waist circumference, body mass index, blood pressure, patients' physical activity level, eating behavior, patients' perception on chronic illness care, satisfaction in patient-physician interaction and perceived absolute 10-year cardiovascular disease risk. Feasibility of implementing the intervention will be evaluated. These include acceptability of the intervention, estimating the likely rate of participant recruitment and retention, appropriateness of the outcome measures, calculation of sample size, and its potential effectiveness.

Conclusion: To our knowledge, this is the first study in Malaysia that aims to determine the feasibility of a multifaceted e-health intervention, as well as to indicate more useful aspects of this intervention for further exploration in a larger trial.

\section{Background}

According to the International Diabetes Federation (IDF) consensus worldwide definition of Metabolic Syndrome (MetS), it is estimated that around $20-25 \%$ of the world's adult population have MetS [1, 2]. The cardiovascular disease (CVD) risk factors tend to cluster in an individual, giving rise to MetS which is defined by the presence of central obesity, elevated blood pressure, elevated plasma glucose, and dyslipidaemia [3]. The prevalence of MetS ranges from $11.9 \%$ to $37.1 \%$ in the Asia-Pacific region based on a systematic review [4]. These include Philippines (11.9\%), China (21.3\%), Sri Lanka (24.3\%), Taiwan (25.5\%), Singapore (26.9\%), South Korea (31.3\%), Mongolia (32.8\%) and Malaysia (37.1\%) [4]. In 
Malaysian adults $\geq 30$ years, the prevalence of MetS was found to be $43.4 \%$ [5]. A recent review showed that MetS affected $25 \%$ to $44 \%$ of the adult population in Malaysia with the risk increasing with age [6].

The prevalence of MetS has reached epidemic proportions in many Asian countries, including Malaysia especially in the younger generations [7, 8]. Rapid economic growth, socio-demographic change and adoption of unhealthy lifestyle that has occurred over the past few decades are thought to be responsible for this rising prevalence [9]. This in turn, has resulted in an upsurge of CVD morbidity and mortality in Malaysia [9]. A recent national report showed that among $95 \%$ of patients presenting with acute coronary syndrome (ACS), $46.2 \%$ had diabetes, $64 \%$ had hypertension and $38.6 \%$ had dyslipidaemia [10]. Malaysians were found to develop ACS at a younger age compared to their Asian and Western counterparts [10]. The mean age of individuals with ACS at admission in Malaysia was 58.6 years old, of which $23.6 \%$ were under the age of 50 years [10].

One of the promising approaches to improve management of MetS in primary care is the integration of the Chronic Care Model (CCM) into the health system. The CCM is the best-known model for transforming chronic disease management in primary care [11]. It focuses on linking informed, activated patients with proactive and prepared health care teams $[11,12]$. CCM offers effective strategies to improve chronic disease outcomes in primary care $[12,13]$. A series of recent studies have indicated that CCM improves the quality of care and outcomes for patients with various chronic conditions [14-16]. In Malaysia, our previous work has shown that it was feasible to implement the CCM in the primary care setting [17]. In the EMPOWER-PAR Study, we have shown that the implementation of at least three components of CCM which include self-management support, improved glycaemic control among patients with Type 2 Diabetes Mellitus [18].

One of the most essential components of the CCM is self-management support which encompasses activities that empower patients to become activated to manage their own health $[11,12]$. International clinical guidelines recommend the inclusion of self-management programs in the management of MetS and the associated cardiovascular (CV) risk factors and such programs have been associated with improved health outcomes $[12,19]$. A systematic review of 19 studies reveals that self-management intervention improved outcomes including haemoglobin A1c (HbA1c), waist circumference (WC), selfefficacy and empowerment in patients with MetS [20]. Lifestyle modification intervention has been shown to be effective in improving fasting blood glucose (FBG), WC, blood pressure (BP), and triglycerides (TG) in patients with MetS [21].

Conceptually, self-management has a close association with patient activation and empowerment [22, 23]. Patient activation reflects the patients' central role in having the knowledge, skills and confidence to make effective decisions to self-manage their own health and change their behaviour e.g. in adopting a healthy lifestyle [23-26]. It is considered to be the most reliable indicator of the willingness and ability to manage health autonomously [27-29]. Previous studies have emphasized that increased patient activation level has been shown to improve self-management behaviours such as physical activity, diet and medication adherence in patients with various chronic conditions [25, 30-32]. Patient activation has 
also been shown to improve quality of life and health outcomes; and to reduce the utilization of health care services and costs for patients with various chronic conditions [25, 30-33]. However, there is a gap in the literature pertaining to intervention targeting to improve patient activation in patients with multiple CVD risk factors such as MetS, especially in the younger generations.

To meet the growing demands of the younger generation, efforts have been made to develop web or mobile applications for self-management of multiple CV risk factors [34-38]. To ensure sustainability, the application should be developed using evidence-based approach such as the persuasive technology [39]. This technology was established to motivate people by influencing attitudes and behaviours of the users through persuasion and social influence, but not through pressure or force [39].

However, to our knowledge, there is no e-health self-management system which has been developed to suit the younger generations with MetS in Malaysia. Therefore, the objective of this study is to evaluate the feasibility and potential effectiveness of the EMPOWER-SUSTAIN Self-Management e-Health Intervention (multifaceted strategies involving web-based self-management mobile apps based on the CCM and persuasive technology theory) in improving patient activation and self-management behaviours compared with usual care among individuals with MetS in the Malaysian primary care setting. This paper describes the design of the trial, the development of the EMPOWER-SUSTAIN Self-Management e-Health Intervention and its underpinning conceptual frameworks.

\section{Hypotheses}

The primary hypothesis is that the mean score of patient activation would improve with the EMPOWERSUSTAIN Self-Management e-Health Intervention. The secondary hypotheses are that WC, body mass index (BMI), BP, physical activity level, eating behaviour, patients' perceptions and experiences of receiving care for chronic conditions, patient-physician satisfaction and the accuracy of the CVD risk perception would improve with the EMPOWER-SUSTAIN Self-Management e-Health Intervention.

\section{Trial design}

This is a pilot randomised controlled trial with parallel group design i.e. intervention vs. control (usual care) with allocation ratio of 1:1. The overall duration of the study is one year, and the duration of the intervention is 6 months. Blinding is not possible due to the nature and complexity of the intervention.

\section{Methods}

\section{Protocol registration}

The study protocol is registered with the ClinicalTrials.gov (identifier: NCT04120779) and the registration complied with all the all items from the World Health Organization Trial Registration Data Set. This is the first version of the study protocol. The reporting of this paper is done in accordance with the SPIRIT 2013 guidance and checklist for protocols of clinical trials [40] and the CONSORT checklist for pilot and 
feasibility trials [41]. The SPIRIT checklist is provided in Additional File 1. Figure 1 shows the EMPOWERSUSTAIN CONSORT Flow Diagram [41].

\section{PLEASE INSERT FIGURE 1 HERE}

\section{Study setting}

This pilot study will be conducted at a university primary care clinic, which is located in the state of Selangor, Malaysia. It is a busy primary care clinic with a load of approximately 500 patients per day. Almost $70 \%$ of the patients are under regular follow-up at this clinic for various long term conditions including MetS.

The pilot randomised controlled trial [42] is conducted to ensure that the intervention can be delivered as intended and safe assumptions can be made about effect size, rate of recruitment and retention in the future definitive clinical trial [43]. In this pilot study, the feasibility of implementing the EMPOWERSUSTAIN ${ }^{\odot}$ Self-Management e-Health Intervention for patients with MetS in a primary care clinic will be evaluated. These include acceptability of the intervention, estimating the likely rate of participant recruitment and retention, appropriateness of the outcome measures, calculation of sample size, and its potential effectiveness i.e. the effect size $[44,45]$.

\section{Study population}

The study population will comprise of individuals aged 18 to 60 years old who are diagnosed with MetS according to the Joint Interim Statement (JIS) on MetS definition, 2009 by the International Diabetes Federation Task Force on Epidemiology and Prevention; National Heart, Lung, and Blood Institute; American Heart Association; World Heart Federation; International Atherosclerosis Society; and International Association for the Study of Obesity [1].

According to the JIS definition [1], MetS is defined by the presence of at least 3 out of 5 of the following risk factors:

I. WC: $M \geq 90 \mathrm{~cm} \mathrm{~F} \geq 80 \mathrm{~cm}$ (South Asian cut-points)

II. BP: Systolic BP $\geq 130$ and/or diastolic BP $\geq 85 \mathrm{mmHg}$ or on treatment for hypertension (HPT)

III. FBG: $\geq 5.6 \mathrm{mmol} / \mathrm{L}$ or on treatment for elevated glucose

IV. TG: $\geq 1.7 \mathrm{mmol} / \mathrm{L}$ or on treatment for TG

V. High density lipoprotein cholesterol (HDL-c): Male \& $1 \mathrm{t} ; 1.0 \mathrm{mmol} / \mathrm{L}$, Female \&lt; $1.3 \mathrm{mmol} / \mathrm{L}$; or on treatment for HDL-C

Patient recruitment 
Consecutive patients who attend the university primary care clinic during the recruitment period will be approached, given the patients information sheet about the study and invited to participate. Those who are willing to participate will be interviewed and screened by the investigators to identify eligibility based on the inclusion and exclusion criteria. Written informed consent will be obtained from those who are eligible and they will be recruited into the study.

\section{Inclusion Criteria}

Patients 18 to 60 years old who fulfil all of the following inclusion criteria will be included:

1. diagnosed with MetS according to the JIS definition [1]

2. have received follow-up care for MetS at the university primary care clinic at least twice in the last one year

3. have regular access to the internet

4. perceive that they have basic skills to use the web and smart mobile phone

5. are able to read and understand written English or Malay

\section{Exclusion Criteria}

Patients who fulfil any of the following criteria will be excluded:

1. type 1 diabetes mellitus

2. receiving renal dialysis

3. present with severe hypertension (systolic BP \&gt; $180 \mathrm{mmHg}$ and/or diastolic BP \&gt; $110 \mathrm{mmHg}$ ) at recruitment

4. diagnosed with conditions resulting in secondary hypertension

5. diagnosed with circulatory disorders requiring referral to secondary care over the last one year and during the course of the study (e.g. unstable angina, heart attack, stroke, transient ischaemic attacks, peripheral vascular diseases)

6. receiving shared care at primary and secondary care centres for CVD (coronary artery diseases, stroke, transient ischaemic attacks, peripheral vascular diseases)

7. receiving chemotherapy/ radiotherapy or palliative care

8. diagnosed with a psychiatric illness such as schizophrenia, bipolar disorder, major depression

9. diagnosed with cognitive impairment such as dementia 
10. pregnant

11. enrolled in another intervention study

\section{Physician recruitment}

All primary care physicians (PCP) who are practicing at the university primary care clinic will be invited to participate in the study. To be eligible, the following criteria must be met:

1. have one or more years of working experience in a primary care setting

2. must be keen to participate in the study

3. willing to deliver the EMPOWER-SUSTAIN ${ }^{\circledR}$ e-Health Intervention to patients with MetS

At least ten PCP who meet the eligibility criteria will be recruited. Five PCP will be allocated to deliver the EMPOWER-SUSTAIN ${ }^{\circledR}$ intervention and the rest of the PCP will continue with usual care.

\section{The intervention}

The EMPOWER-SUSTAIN ${ }^{\circledR}$ Self-Management e-Health Intervention is a complex intervention involving multifaceted components using CCM as the conceptual framework [11, 12]. This is in line with the United Kingdom (UK) Medical Research Council (MRC) complex intervention framework, which recommends that intervention development be guided by best available evidence and appropriate framework [46, 47]. A comprehensive review of the literature was conducted to identify the crucial components of the EMPOWER-SUSTAIN ${ }^{\circledR}$ intervention that could improve patient activation and self-management behaviours. Three crucial components were identified based on the 3 CCM elements i.e. delivery system design, self-management support and decision support; and will be included in the EMPOWER-SUSTAIN ${ }^{\circledR}$ Self-Management e-Health Intervention as described in Table 1. Figure 2 illustrates the conceptual framework underpinning the EMPOWER-SUSTAIN ${ }^{\circledR}$ Self-Management e-Health Intervention.

\section{PLEASE INSERT TABLE 1 HERE}

\section{PLEASE INSERT FIGURE 2 HERE}

\section{Development of the EMPOWER-SUSTAIN self-management mobile application ${ }^{\odot}$}

The EMPOWER-SUSTAIN web-based self-management mobile application ${ }^{\circledR}$ is currently at the final stage of development. Iterative Model was chosen as the software development model for this study [48]. Content from the newly revised EMPOWER-SUSTAIN Global Cardiovascular Risks Self-Management Booklet $^{\circledR}$ was evaluated for its suitability to be included in the prototype. A storyboard was designed to create the flow of prototype usage by PCP, nurse and patients during follow-up clinic and at home. In the pre-alpha stage, wireframe was designed to describe and visualize the user interface in static draft 
layouts based on the content and structure of information. Based on the wireframe, high-fidelity mock-up static graphic diagrams demonstrating the content and function of the prototype divided into eight sections were designed. The sections included My Profile, My Cardiovascular Risks, My Treatment Targets, My Check-Up, My Weight Management, My Smoking Habit, My Self-Management and My Medication. Persuasive technology theory $[39,49]$ was used to build the evidence-based content which is presented in a user friendly interface, with incorporation of interactivity and reward system. This app is designed to aid and motivate people to adopt positive attitude and behaviour change through persuasion and reward system. The EMPOWER-SUSTAIN mock prototype mobile app ${ }^{\odot}$ is provided in Additional File 2. Based on the mock graphic diagrams, a working prototype of the EMPOWER-SUSTAIN self-

management mobile app ${ }^{\odot}$ was developed using the iterative model of software development life cycle (SDLC) $[48,50]$. A complementary desktop application is also being developed for the PCP and nurse to use in the clinic. This prototype is currently undergoing the alpha (utility) testing by medical experts [51, 52] and beta (usability) testing by patients with MetS [53]. Data which are entered by the PCP and patients into the app will be stored in a secure server. The feasibility of using the app will be evaluated in this pilot study.

\section{Conduct of the EMPOWER-SUSTAIN ${ }^{\circ}$ Workshop}

Prior to the delivery of intervention, the five PCP in the intervention arm and a nurse will be trained on how to utilise the EMPOWER-SUSTAIN ${ }^{\odot}$ desktop and mobile applications during the EMPOWER-SUSTAIN ${ }^{\odot}$ Workshop. Training will also include reinforcing the use of relevant clinical practice guidelines (CPG) [54] as decision support for management and prescribing. The PCP and nurse will be trained based on the Self-Determination Theory (SDT) which would be useful for them to understand how patients' behaviour change can be influenced by healthcare providers [55]. They will also be trained on motivational interview (MI) techniques and health coaching skills to facilitate patients' autonomous self-regulation to enhance their behaviour change [55-58]. Ml training would include the skills to formulate open-ended questions, reflective statements (i.e. restating what the patients conveyed) and stimulating what the patients know before providing relevant education [55]. The main aim of the $\mathrm{MI}$ is to engage patients in problem solving by encouraging active learning [56] and facilitating patients' autonomous self-regulation to achieve goals by enabling patients to develop individualised action plans $[57,58]$.

\section{Delivery of the intervention}

The EMPOWER-SUSTAIN ${ }^{\odot}$ Self-Management e-Health Intervention will be professionally delivered to the individual patients by the PCP, assisted by a nurse. Patients will be given the EMPOWER-SUSTAIN selfmanagement $\mathrm{app}^{\odot}$ which will be downloaded to their mobile phone. Follow-up care by the PCP will be arranged at baseline, 3-month and 6-month. At baseline, patients in the intervention arm will be given a username and password to access the app. They will be trained individually on its utilisation by the PCP assisted by a nurse using the Knowledge to Action (KTA) framework [59] and the persuasive technology theory $[39,49]$. The KTA framework incorporates the need to adapt the knowledge to fit with individual context. In addition to this, PCP and the nurse will provide problem-solving assistance and support for 
patients to use the app in line with the theory of persuasive technology. MI techniques and health coaching skills to facilitate patients' autonomous self-regulation to enhance their behaviour change will also be applied. PCP and the nurse will go through each section with the patients and will ensure that information recorded in all sections is complete. Patients will be guided to navigate through the functions of the mobile app to ensure competence, especially on the sections involving self-management. Patients will be coached to use the app and to review their progress at home e.g. self-monitoring of their weight, BP and blood glucose, and recording of their physical activity and diet. Particular attention will be given

to specific pages of the app which summarise patients' achievement in their self-management goals and clinical outcomes. These achievements will be rewarded with star rating in accordance with the persuasive technology theory. PCP and the nurse will discuss self-management progress and goals using the app with the patients at each follow-up visit. The goal of the intervention is to improve patient activation and self-management behaviours through patient-physician collaboration and regular reinforcement to ensure sustainability. Figure 3 illustrates the delivery of the EMPOWER-SUSTAIN ${ }^{\odot}$ SelfManagement e-Health Intervention.

\section{PLEASE INSERT FIGURE 3 HERE}

\section{Monitoring the intervention}

During the 6-month intervention period, patients are required to utilize the EMPOWER-SUSTAIN selfmanagement mobile $\mathrm{app}^{\odot}$ for a cumulative period of two hours duration, and be seen at least once by the PCP for follow-up care. A separate web interface will be created for PCP to monitor patients' log-in frequency and duration of utilisation of the tool. Patients who do not comply with the utilisation and follow-up requirements will be considered as lost to follow-up. Patients who are lost to follow-up or who withdraw from the trial will not be replaced. Analysis will be by intention to treat. There is no other specific concomitant care and intervention that is permitted nor prohibited during the trial.

\section{Outcome measures}

Outcome measures are divided into primary and secondary outcomes. These measures will be obtained from both intervention and control groups at baseline and 6-month after the delivery of the intervention.

\section{Primary outcome}

The primary outcome will be measured by the mean change in patient activation score using the Patient Activation Measure ${ }^{\circledR}$ short form Malay version (PAM $\left.{ }^{\circledR}-13-\mathrm{M}\right)$ questionnaire [24].

\section{Secondary outcomes}

1. Change in the mean WC

2. Change in the mean BMI 
3. Change in the mean systolic and mean diastolic BP

4. Change in the physical activity level will be measured by the International Physical Activity Questionnaire short form - Malay version (IPAQ-M) [60].

5. Change in the eating behaviour will be measured by the Dutch Eating Behaviour Questionnaire - Malay version (DEBQ-M) [61].

6. Change in the patients' perceptions and experiences of receiving care for chronic conditions will be measured by the Patients Assessment of Chronic Illness Care - Malay version (PACIC-M) questionnaire [62].

7. Change in the patient-physician satisfaction will be measured by the Skala Kepuasan Interaksi Perubatan (SKIP-11) questionnaire [63].

8. Change in the accuracy of CVD risk perception will be measured by the absolute difference between the perceived absolute 10-year CV risk and the actual calculated risk by Framingham Risk Score (FRS) General CVD risk prediction chart [64-66].

\section{Control group}

The control group will continue to receive usual care at the university primary care clinic. They will be given the EMPOWER-SUSTAIN Global CV Risks Self-Management Booklet ${ }^{\circledR}$, as this is considered as usual care at the university primary care clinic. The EMPOWER-SUSTAIN self-management mobile app ${ }^{\odot}$ will be made available to the control group at the end of the study. During the course of the study, there will be no limit to the number of clinic visits that a patient is allowed to make in either the intervention or control groups.

Figure 4 shows the EMPOWER-SUSTAIN ${ }^{\odot}$ schedule of enrolment, interventions and assessments according to SPIRIT guideline [40].

\section{PLEASE INSERT FIGURE 4 HERE}

\section{Study procedures}

All interviewers and investigators will be trained regarding the study procedures prior to the conduct of the study to minimise variability in the method of data collection. Data will be collected at baseline and 6 months after delivery of the intervention. The study procedures will be conducted in accordance with the Malaysian Guideline for Good Clinical Practice, 4th edition, 2018 [67].

\section{Demographic and anthropometric data collection}

A standardised case report form (CRF) will be used to collect socio-demographic information of the patients (age, gender, ethnicity, patients contact details, education attainment, and occupation), smoking 
status (including the number of cigarettes smoked per day for current smokers) and other clinical information (presence of comorbidities, past medical history and family history). Data on pharmacological treatment will be systematically collected from the medical records of the study patients using CRF at baseline and at 6-month follow-up in both the intervention and the control clinics.

Height and weight will be measured using the Seca 769 Digital Medical Scale stadiometer. Weight will be measured in light clothing, without shoes on the scale with a precision of $0.1 \mathrm{~kg}$. Height will be measured to $0.1 \mathrm{~cm}$ using the stretch stature method of the stadiometer and then converted to metres. BMI will be calculated using the standard formula (weight in $\mathrm{kg}$ )/ (height in metres) ${ }^{2}$.

WC will be measured to the nearest $0.1 \mathrm{~cm}$ using non-stretchable measuring tape with the patients standing in a relaxed position and arms at the side. The measurement will be taken at the midpoint between the lower rib margin (12th rib) and the top of the iliac crest in horizontal plane. Each measurement will be repeated twice. If the measurements are within $1 \mathrm{~cm}$ of one another, the average will be calculated. If the difference between the two measurements exceeds $1 \mathrm{~cm}$, both measurements will be repeated.

Patients will be made to rest and will not be allowed to eat, drink caffeinated beverages, exercise, smoke cigarettes or climb stairs for at least 15 minutes before BP measurements are taken. BP will be measured twice, two minutes apart on the right arm in a sitting position, using an Omron IA2 model automatic digital blood pressure monitor. Each patient will be seated upright with his/her right arm supported at the heart level. The mean of the first and second systolic and diastolic measurements will be reported as the $B P$ value for individual patients if the difference is within $5 \mathrm{mmHg}$. If the readings differ by more than $5 \mathrm{mmHg}$, the measurements will be repeated.

\section{Administration of the questionnaires}

Patients in both the intervention and control arms will be given a set of questionnaires to be selfadministered which include PAM-13-M, IPAQ-M, DEBQ-M, PACIC-M, SKIP-11 and a visual analogue scale to record the perceived absolute 10-year CVD risk.

Clear written and verbal instructions will be given on how to fill up the questionnaires. They will be requested to circle or tick which options suited them the most. Patients will be encouraged to seek clarification from the investigators at any time should any queries arise. They will also be reminded to answer the questionnaires themselves rather than getting help from their accompanying family members.

Patients will be given a pen to complete the questionnaires at a corner of the clinic equipped with tables and chairs. The investigators will ensure that patients do not interact with each other whilst answering the questionnaires. On average, patients will be expected to take approximately 30 minutes to complete the set of questionnaires. Once they have finished, they will hand the questionnaires to the investigators, who will then check the responses for completeness. 


\section{Study tools}

\section{Patient Activation Measure ${ }^{\circledR} 13$ - Malay version $\left(P A M^{\circledR}-13-M\right)$ questionnaire}

The PAM ${ }^{\circledR}-13$ consists of 13 items measuring patients' self-reported knowledge, skills and confidence for self-management [24]. It was developed in the English language using a Rasch model [24]. Each item has five response categories with scores from 1 to 5: (1) "Strongly Disagree", (2) "Disagree", (3) "Agree", (4) "Strongly Agree" and (5) "Not Applicable". The instrument design reflects the four stages of activation in a progressing difficulty of the items: level 1 (patients believe that their role is important: item 1 and 2), level 2 (patients have confidence and knowledge to take action: items 3-8), level 3 (taking action: items 9-11) and level 4 (staying on course under stress: items 12 and 13). According to the PAM $^{\circledR}-13$ scoring guidelines, the raw scores are transformed through natural logarithm to achieve a better expression of the relative distance between the scores. Then, items are transformed to a standardized metric ranging from 0 to 100 ( 0 = lower activation; $100=$ highest activation). The score is calculated by summing up the raw scores and mapping up the sum onto a scale of $0-100$. A higher score of PAM ${ }^{\circledR}-13$ indicates a high level of patient activation [24]. The $P A M^{\circledR}-13$ is one of the most extensively used, widely translated and tested instrument worldwide in measuring patient activation level in self-management. It has been translated into the Malay language and is currently being validated in the study population. Research licence to utilize this questionnaire will be obtained from Insignia Health ${ }^{\circledR}$, University of Oregon.

\section{International Physical Activity Questionnaire short form - Malay version (IPAQ-M)}

The IPAQ was developed to measure health-related physical activity (PA) in the English language [68] and was previously translated into the Malay language and validated in the Malaysian population [60]. The IPAQ-M short version comprises of 12 items, covering vigorous, moderate, walking, sitting and sleeping activities [60]. Patients are required to report the activities performed during the last seven days and to include only activities that lasted 10 minutes or more per session. IPAQ will be scored according to its scoring protocol [69]. Continuous score will be expressed as Metabolic Equivalent Task (MET)-minutes per week: MET level $x$ minutes of activity/day $x$ days per week [69]. The scores will then be categorised into 'Low', 'Moderate' and 'Vigorous' physical activity levels, in accordance to the Malaysian CPG on Primary and Secondary Prevention of Cardiovascular Disease 2017 [54]. The IPAQ-M short version has acceptable validity for moderate, vigorous and total physical activity, and was found to be reliable for assessing physical activities of Malay adults [60].

\section{Dutch Eating Behaviour Questionnaire-Malay version (DEBQ-M)}

The DEBQ was developed in the English language [70] and has been translated into the Malay language and validated in the Malaysian population [61]. The DEBQ-M contains 33 items to measure emotional, external, and restrained eating behaviours. Emotional eating is assessed by 13 items, whereas external and restrained eating behaviours are assessed by 10 items each. The questions that assess the three different behaviours appear in random order in the questionnaire and are answered according to the 
Likert scale with a scoring system identified as follows: 1 = never, 2 = rarely, $3=$ sometimes, $4=$ often, and $5=$ very often. There are three sub-scales in the instrument. For each subscale, the score is added and divided by the number of items in the sub-scale to obtain the average score for emotional, external and restrained eating for a person [61].

\section{Patients Assessment of Chronic IIIness Care - Malay version (PACIC-M) questionnaire}

The PACIC questionnaire consists of 20-item patient self-report instrument developed in the English language to assess the extent to which patients with chronic disease receive care that aligns with the CCM [71]. It has recently been translated into the Malay language, cross-culturally adapted and validated to produce the PACIC-M [62]. It measures care that is patient-centred, proactive and planned, which includes collaborative goal setting, problem-solving and follow-up support [71]. Each item is scored on a 5 -point Likert scale with 1 being 'no' or 'never' and 5 being 'yes' or 'always'. The higher the score, the more aligned is the perceived care to CCM. PACIC-M was found to be highly reliable (Cronbach's a of 0.94 with mean inter-item correlation of 0.45 ) and valid to be used in assessing 3 CCM model domains [62].

\section{Skala Kepuasan Interaksi Perubatan-11 (SKIP-11) Questionnaire}

SKIP-11 is the translated and validated Malay version [63] of the Medical Interview Satisfaction Scale (MISS-21) [72]. SKIPP-11 is used to measure patient-physician interaction satisfaction and consists of 11 questions representing three subdomains of patient-physician interaction satisfaction. There are four questions pertaining to information provision ("Distress relief" subdomain), four questions regarding the physician's communication skills ("Rapport" subdomain) and three questions assessing the adherence intent as an outcome of the overall interaction experience ("Interaction outcome" subdomain). All 11 items are scored using a 5-point Likert scale whereby for positively worded items, score ' 5 ' is for 'strongly agree' and score ' 1 'is for 'strongly disagree'. For the negatively worded items, score 1 is for 'strongly agree'

and score ' 5 ' is for strongly disagree. Each response will be added together to give a total score within the range of 11 (minimum) and 55 (maximum). Total score for each subdomain is also calculated and analysed where the minimum and maximum score is determined by the number of items present in each subdomain. The levels of satisfaction will be determined by the proximity of the score to either the minimum or maximum score for each subdomain. The closer proximity of the score to the maximum score will reflect good satisfaction level and vice versa [63].

\section{Accuracy of the Perceived Absolute 10-year CVD Risk}

The perceived absolute 10-year risk of heart attack and stroke will be estimated separately by the patients along a visual analogue scale [64]. The average of these values will be taken as the perceived absolute 10-year CVD risk for the patients. The patients' actual absolute 10-year CVD risk will be calculated using the FRS General CVD risk prediction chart $[65,66]$, which has been validated in the Malaysian population [73]. The accuracy of the CVD risk perception will be defined as the absolute difference between the perceived and the actual risk, which will be inversely related i.e. the lower the absolute difference, the more accurate the patients' CVD risk perception [64]. 


\section{Sample size determination}

Sample size is calculated using Power and Sample Size Calculation software version 3.1.2, 2014 [74] based on the findings of a randomised controlled trial evaluating the effects of a web-based selfmanagement intervention for adults with chronic conditions on patient activation scores, measured by the $\mathrm{PAM}^{\circledR}-13$ questionnaire [75]. In the intervention group, the mean patient activation score at baseline was 65.33 and the mean score after the intervention was 71.30 (mean difference of $5.97 \pm 9.70, t 57=$ $4.683, P$ \& lt; 0.001) [75]. Whereas, in the control group, the mean patient activation score at baseline was 66.89 and the mean score at the end of the study period was 68.93 (mean difference of $2.04 \pm 10.01$, t67 $=1.677, \mathrm{P}=0.10$ ) [75]. Therefore, the mean difference between the two groups was 3.93 .

Based on this assumption, a sample size of 97 patients per group is sufficient to detect mean difference of $\delta=3.93$ in the patient activation score between the two groups, with a standard deviation of $\sigma=9.70$ using two-tailed t-test of difference between means with $80 \%$ power (power $=0.8$ ), $5 \%$ level of significance $(a=0.05)$ and sample size ratio of $1: 1$ between the two groups $(m=1)$. After considering a drop-out rate of $20 \%$, the sample size required is 116 patients per group, giving a total of 232 patients to be recruited for this study.

\section{Randomisation}

Randomisation of patients to either the EMPOWER-SUSTAIN ${ }^{\circledR}$ Self-Management e-Health Intervention (I) or usual care (C) will be done using Randomised Block Design by a research assistant. Random allocation will be made in order to keep the sizes of the group similar. In this study, the block randomisation size will be 4 times the number of treatment arms i.e. block size of 2 by 2 . With two treatment arms of the EMPOWER-SUSTAIN Self-Management e-Health Intervention (I) or usual care (C), the possible treatment allocations within each block [76] will be as follows: IICC, ICIC, ICCl, CCII, CICl, CIIC. A research assistant will generate the allocation sequence, enrol the participants, and will assign the patients to the intervention or control arms according the sequence. The generated sequence will be placed in sealed envelopes to ensure that it is concealed from the PCP until the intervention is assigned. Blinding is not possible due to the complexity of the intervention.

\section{Data management}

Each CRF will be given a unique identifier. Data collected using the CRF and all the questionnaires will be checked by a research assistant to ensure completeness. If any missing data is found, the patients will be contacted again via telephone. Double data entry into SPSS version 24 will be conducted and data cleaning will be done to manage outliers, missing values and inconsistencies. The clean datasets for baseline and outcome will be used for analysis. Data will be stored in a secure database at the Institute of Pathology, Laboratory and Forensic Medicine (I-PPerForM), Universiti Teknologi MARA (UiTM).

\section{Statistical analysis}


The analysis will be conducted using SPSS version 24.

\section{Descriptive analysis}

Frequency distribution, measure of central tendency and dispersion will be produced. For the continuous data, it will be presented by mean and standard deviation or median based on the normality of the data. For the categorical data, it will be presented by absolute number and their corresponding percentages.

\section{Effectiveness analysis}

Intention to Treat (ITT) analysis will be applied to measure the potential effectiveness of the EMPOWERSUSTAIN $^{\odot}$ Self-Management e-Health Intervention on the primary and secondary outcome measures based on the initial treatment assignment. The mixed model repeated measure analysis of variance (ANOVA) will be carried out to evaluate the potential effectiveness i.e. to compare the mean changes in patient activation, physical activity, diet, patients' chronic illness care and patient-physician interaction satisfaction scores, perceived absolute 10-year CVD risk within and between the intervention and control groups at baseline and 6-month follow-up.

\section{Feasibility outcomes}

Process evaluation to assess the integrity of the randomised controlled trial protocol will be conducted. These include recruitment rate, methods of randomisation, retention rate, appropriateness of the primary and secondary outcome measures, sample size calculation, whether the intervention could be delivered as intended and methods of statistical analysis to evaluate the potential effectiveness. Qualitative studies to explore facilitators and barriers in delivering the intervention among PCP, and utilising the intervention among patients will also be conducted. However, detailed method for the qualitative studies is beyond the scope of this paper.

\section{Data monitoring}

Data monitoring will be done by the EMPOWER-SUSTAIN ${ }^{\odot}$ investigators. Data on any adverse event, unintended effect of trial intervention or trial conduct will be collected, assessed, reported and managed by the investigators. External data monitoring committee is not needed as the intervention does not involve new pharmacological agent or regulated device.

\section{Discussion}

To the best of our knowledge, the EMPOWER-SUSTAIN ${ }^{\odot}$ project is the first self-management e-health intervention designed for patients with MetS in the Malaysian primary care setting. The pilot randomised controlled trial will be conducted to evaluate the feasibility and potential effectiveness of the EMPOWERSUSTAIN ${ }^{\odot}$ intervention. These include recruitment rate, methods of randomisation, retention rate, selection of primary and secondary outcome measures, sample size calculation, whether the intervention 
could be delivered as intended and methods of statistical analysis to evaluate the potential effectiveness $[44,45]$. All of these aspects will be useful for further exploration in a larger definitive trial.

The EMPOWER-SUSTAIN ${ }^{\odot}$ Self-Management e-Health Intervention is expected to yield important new evidence on the potential improvements of patient activation and self-management behaviours among patients with MetS in a developing country. It is hypothesised that patients' activation, waist circumference, BMI, blood pressure, physical activity level, eating behaviour, perception and experience of receiving chronic disease care, patient-physician satisfaction and perceived absolute 10-year CVD risk would improve with the EMPOWER-SUSTAIN ${ }^{\odot}$ Self-Management e-Health Intervention.

The EMPOWER-SUSTAIN ${ }^{\circledR}$ intervention is a complex, multifaceted chronic disease management strategy based on $3 \mathrm{CCM}$ elements i.e. delivery system design, self-management support and decision support. It consists of training PCP, nurse and patients to use the EMPOWER-SUSTAIN web-based self-management mobile app ${ }^{\odot}$, strengthening patient-physician relationship and reinforcing the use of relevant CPG for management and prescribing. This intervention is developed based on the MRC UK recommendations, guided by the best available evidence and appropriate framework $[46,47]$.

It is well established that the CCM is one of the best-known models to transform chronic disease care [11]. The EMPOWER-SUSTAIN ${ }^{\odot}$ intervention focuses on linking informed, activated patients with proactive and prepared practice team i.e. PCP and nurse $[11,77]$. The self-management mobile app is developed as a tool for health care providers to support and engage patients so that they are empowered with knowledge, skills and confidence to take independent actions to manage their own health $[11,12]$.

Development of an e-health intervention to support patients' self-management requires careful planning and the use of theory-based strategies to increase the probability of effectiveness, programme adoption and implementation [34-37]. Therefore, the EMPOWER-SUSTAIN self-management mobile app ${ }^{\odot}$ is developed using the persuasive technology theory [39]. It consists of evidence-based content presented in a user friendly interface, with incorporation of interactivity, reward system, problem-solving assistance, patient-physician collaboration and regular reinforcement to ensure sustainability [34-37, 78-81]. It is designed to aid and motivate people to adopt positive attitude and behaviour change through persuasion and social influence [39].

To ensure effective patient-physician collaboration, PCP will be trained prior to the delivery of intervention. In the EMPOWER-SUSTAIN ${ }^{\odot}$ Workshop, PCP and a nurse will be trained based on the SDT, an evidence-based framework of human motivation and personality theory which supports the individual's experience of autonomy, competence, relatedness and engagement for activities [55]. It is useful for them to understand that individual's behaviour change can be influenced by the role of a healthcare provider [55]. They will also be trained on Ml techniques and health coaching skills to facilitate patients' autonomous self-regulation to enhance their behaviour change by enabling patients to develop individualised action plans [55-58]. 
PCP assisted by a nurse will then train individual patients on how to utilise the EMPOWER-SUSTAIN selfmanagement mobile app ${ }^{\odot}$ based on the KTA framework [59]. This framework is chosen because it is context-focused, enables knowledge-producer and knowledge-user collaboration, and emphasizes sustainability [59]. The KTA framework incorporates the need to adapt the knowledge to fit with individual context. This framework is particularly useful for emphasizing the collaboration between knowledge producers and knowledge users [59]. Sustainable knowledge use is essential given the chronic nature of MetS and the associated CV risk factors. Apart from the KTA framework, the PCP and nurse will also apply the $\mathrm{Ml}$ techniques and health coaching skills to influence behaviour change in these patients. $\mathrm{A}$ systematic review on the effectiveness of $\mathrm{Ml}$ in primary care has reported positive outcome of this intervention on health behaviour change [82].

Another essential component of the CCM is clinical decision support [11]. Clinical management should be tailored towards individual patients' need guided by evidence-based decision support tool i.e. the CPG [11]. Therefore, PCP will be trained to utilise the relevant evidence-based CPG to support management and prescribing for patients with MetS. This would empower PCP to improve their clinical management [83].

\section{Conclusion}

Ultimately, the results from this pilot study will determine the feasibility of this multifaceted e-health intervention, as well as to indicate more useful aspects of this intervention for further exploration in a larger trial. This study will also provide the evidence of potential effectiveness of a multifaceted intervention involving web-based self-management mobile app, which is developed based on the CCM and persuasive technology theory in primary care setting. It is hoped that the evidence from this study will provide a platform to support larger definitive clinical trial to evaluate the effectiveness of this e-health self-management intervention in Malaysia.

\section{Trial Status}

This study is at the final stage of development of the EMPOWER-SUSTAIN self-management mobile $\mathrm{app}^{\odot}$. Participants screening and recruitment is expected to commence in March 2020. The expected date of completion of patient recruitment is April 2020. Baseline data collection is planned to start in MayJune 2020. The intervention is planned to be delivered for 6 months from July to December 2020. The outcome data will be collected in January-February 2021. The expected date of completion of this pilot trial is $30^{\text {th }}$ June 2021.

\section{List Of Abbreviations}

MetS $=$ Metabolic syndrome

$\mathrm{CCM}=$ Chronic Care Model 
$\mathrm{CPG}=$ Clinical practice guidelines

CVD = Cardiovascular disease

IDF = International Diabetes Federation

ACS $=$ Acute coronary syndrome

$\mathrm{HbA1c}=$ Haemoglobin A1c

WC $=$ Waist circumference

FBG = Fasting Blood Glucose

$\mathrm{BP}=$ Blood pressure

$\mathrm{TG}=$ Triglycerides

$\mathrm{CV}=$ Cardiovascular

MRC UK = Medical Research Council, United Kingdom

JIS = Joint Interim Statement

HPT = Hypertension

HDL-c = High Density Lipoprotein Cholesterol

$\mathrm{PCP}=$ Primary care physicians

SDLC = Software development life cycle

SDT $=$ Self-Determination Theory

$\mathrm{MI}=$ Motivational Interview

$\mathrm{KTA}=$ Knowledge to Action

$\mathrm{PAM}^{\circledR}-13-\mathrm{M}=$ Patient Activation Measure ${ }^{\circledR}$ short form Malay version

IPAQ-M = International Physical Activity Questionnaire Malay version

MET = Metabolic Equivalent Task

DEBQ-M = Dutch Eating Behaviour Questionnaire - Malay version

PACIC-M = Patients Assessment of Chronic Illness Care - Malay version 
SKIP-11 = Skala Kepuasan Interaksi Perubatan-11

FRS $=$ Framingham Risk Score

$\mathrm{CRF}=$ Case report form

$\mathrm{BMI}=$ Body Mass Index

PA = Physical activity

$\mathrm{MOH}=$ Ministry of Health

$\mathrm{SD}=$ Standard Deviation

ITT = Intention to Treat

\section{Declarations}

\section{Ethics approval and consent to participate}

This study protocol has already been approved by the Research Ethics Committee (REC) of Universiti Teknologi MARA [600-IRMI (5/1/6)/REC/134/19]. This study will be conducted in accordance with the Declaration of Helsinki and Good Clinical Practice (GCP) requirements (Ministry of Health, 2011). The REC will be informed if there is any amendment made to study protocol. Information sheets for patients and physician will be distributed and written informed consent will be obtained from both patients and physicians prior to the enrolment. A research assistant will be trained by the investigators to conduct these procedures. Patients' enrolment will be done by the research assistant and not the patients' attending doctors to reduce patients' perceived coercion to participate in the study. The EMPOWERSUSTAIN consent forms for the patients and physicians are provided as Additional Files $\mathbf{3}$ and $\mathbf{4}$, respectively.Patients will be informed of any immediate results obtained from the study that might affect their care or health. Confidentiality of personal information will be ensured at all times by keeping the data in a password protected secured database at I-PPerForM, UiTM. Research license to use the $\mathrm{PAM}^{\circledR}-13-\mathrm{M}$ will be obtained from Insignia Health ${ }^{\circledR}$, University of Oregon prior to the baseline data collection.

\section{Consent for publication}

Patients' consent for publication is not applicable as patients' individual data will neither be provided nor presented in the manuscript.

\section{Availability of data and material}

Data will be kept at the Institute of Pathology, Laboratory and Forensic Medicine (I-PPerForM), Universiti Teknologi MARA (UiTM), Sungai Buloh Campus, Jalan Hospital, 47000 Sungai Buloh, Selangor, Malaysia. 
Data can be shared upon request and is subjected to the data protection regulations.

\section{Competing interests}

The authors declare that they have no competing interests.

\section{Funding}

This work is funded by the Ministry of Education (MOE), Malaysia: Prototype Research Grant Scheme (PRGS) no: PRGS/1/2018/SKK05/UiTM/01/2 or 600-IRMI/PRGS 5/3 (003/2019). The funding body does not play any role in the design of the study, or in data collection, analysis or interpretation, or in writing the manuscript.

\section{Authors' contributions}

MHD and ASR conceptualised and designed the study. ASR acquired the funding and coordinated the study. MHD and ASR drafted the manuscript and revised it critically for important intellectual content. FHY and NJ developed the mobile app. SAR, RMI, FHY, NB, MSMY, SFBS, AWN, NJ and HMN made substantial critical contributions to the study protocol and the manuscript. All authors have read and given approval of the final manuscript.

\section{Acknowledgements}

The authors wish to thank Fatin Amili Zamros Yuzadi for contributing the original drawings as featured in Figure 2 and 3.

\section{Dissemination policy}

The EMPOWER-SUSTAIN ${ }^{\circledR}$ investigators will prepare and submit progress report of this trial to the funding bodies i.e. the MOE and UITM. Investigators will also publish the outcomes of this pilot and feasibility study including negative outcomes (if any).

\section{References}

1. Alberti KGMM, Eckel RH, Grundy SM, Zimmet PZ, Cleeman JI, Donato KA, et al. Harmonizing the metabolic syndrome: a joint interim statement of the International Diabetes Federation Task Force on Epidemiology and Prevention; National Heart, Lung, and Blood Institute; American Heart Association; World Heart Federation; International Atherosclerosis Society; and International Association for the Study of Obesity. Circulation. 2009;120:1640-5. doi: 10.1161/CIRCULATIONAHA.109.192644.

2. Saklayen MG. The Global Epidemic of the Metabolic Syndrome. Curr Hypertens Rep. 2018;20:12. doi: 10.1007/s11906-018-0812-z. 
3. O'Neill S, O'Driscoll L. Metabolic syndrome: a closer look at the growing epidemic and its associated pathologies. Obes Rev. 2015;16:1-12. doi: 10.1111/obr.12229.

4. Ranasinghe $P$, Mathangasinghe $Y$, Jayawardena R, Hills AP, Misra A. Prevalence and trends of metabolic syndrome among adults in the asia-pacific region: a systematic review. BMC Public Health. 2017;17:101. doi: 10.1186/s12889-017-4041-1.

5. Ramli AS, Daher AM, Nor-Ashikin MN, Mat-Nasir N, Ng KK, Miskan M, et al. JIS definition identified more Malaysian adults with metabolic syndrome compared to the NCEP-ATP III and IDF criteria. Biomed Res Int. 2013;2013:760963. doi: 10.1155/2013/760963.

6. Lim KG, Cheah WK. A Review of Metabolic Syndrome Research in Malaysia. Med J Malaysia. 2016;71:20-8.

7. Ueshima H, Sekikawa A, Miura K, Turin TC, Takashima N, Kita Y, et al. Cardiovascular disease and risk factors in Asia: a selected review. Circulation. 2008;118:2702-9. doi:

10.1161/CIRCULATIONAHA.108.790048.

8. Institute for Public Health (IPH). National Health and Morbidity Survey (NHMS) 2015 vol. II: NonCommunicable Diseases, Risk Factors \& Other Health Problems. in National Health and Morbidity Survey 2015, Institute for Public Health, National Institutes of Health Ministry of Health Malaysia.

9. Azizi A, Rafidah HM. Prevalence and Clinical Characteristics of Metabolic Syndrome among Malaysian Hypertensive Subjects using the International Diabetes Federation Definition. International Medical Journal Malaysia. 2017;16:3-9.

10. Ahmad WAW. National Cardiovascular Disease Database (NCVD): Annual Report of the Acute Coronary Syndrome (ACS) Registry 2014 - 2015. National Heart Association of Malaysia (NHAM) and Ministry of Health Malaysia. 2017.

11. Wagner EH, Austin BT, Davis C, Hindmarsh M, Schaefer J, Bonomi A. Improving chronic illness care: translating evidence into action. Health Aff (Millwood). 2001;20:64-78. doi: 10.1377/hlthaff.20.6.64.

12. Bodenheimer T, Lorig K, Holman H, Grumbach K. Patient Self-management of Chronic Disease in Primary Care. Journal American Medical Association. 2002;288.

13. Si D, Bailie R, Weeramanthri T. Effectiveness of chronic care model-oriented interventions to improve quality of diabetes care: a systematic review. Primary Health Care Research \& Development. 2008;9. doi: $10.1017 /$ s1463423607000473.

14. Davy C, Bleasel J, Liu H, Tchan M, Ponniah S, Brown A. Effectiveness of chronic care models: opportunities for improving healthcare practice and health outcomes: a systematic review. BMC Health Serv Res. 2015;15:194. doi: 10.1186/s12913-015-0854-8. 
15. Bongaerts BW, Mussig K, Wens J, Lang C, Schwarz P, Roden M, et al. Effectiveness of chronic care models for the management of type 2 diabetes mellitus in Europe: a systematic review and metaanalysis. BMJ Open. 2017;7:1-16. doi: 10.1136/bmjopen-2016-013076.

16. Reynolds R, Dennis S, Hasan I, Slewa J, Chen W, Tian D, et al. A systematic review of chronic disease management interventions in primary care. BMC Fam Pract. 2018;19:11. doi: 10.1186/s12875-017-06923.

17. Ariffin F, Ramli AS, Daud MH, Haniff J, Abdul-Razak S, Selvarajah S, et al. Feasibility of Implementing Chronic Care Model in the Malaysian Public Primary Care Setting. Med J Malaysia. 2017;72:106-12.

18. Ramli AS, Selvarajah S, Daud MH, Haniff J, Abdul-Razak S, Tg-Abu-Bakar-Sidik TM, et al. Effectiveness of the EMPOWER-PAR Intervention in improving clinical outcomes of type 2 diabetes mellitus in primary care: a pragmatic cluster randomised controlled trial. BMC Fam Pract. 2016;17:1-18. doi: 10.1186/s12875-016-0557-1.

19. Kennedy A, Reeves D, Bower P, Lee V, Middleton E, Richardson G, et al. The effectiveness and cost effectiveness of a national lay-led self care support programme for patients with long-term conditions: a pragmatic randomised controlled trial. J Epidemiol Community Health. 2007;61:254-61. doi: 10.1136/jech.2006.053538.

20. Kuo CC, Lin CC, Tsai FM. Effectiveness of Empowerment-Based Self-Management Interventions on Patients with Chronic Metabolic Diseases: A Systematic Review and Meta-Analysis. J Adv Nursing. 2014;11:301-15. doi: 10.1111/jan.13574.

21. Yamaoka K, Tango T. Effects of lifestyle modification on metabolic syndrome: a systematic review and meta-analysis. BMC Medicine. 2012;10:138.

22. Bravo P, Edwards A, Barr PJ, Scholl I, Elwyn G, McAllister M, et al. Conceptualising patient empowerment: a mixed methods study. BMC Health Serv Res. 2015;15:252. doi: 10.1186/s12913-0150907-z.

23. Hibbard JH, Stockard J, Mahoney ER, Tusler M. Development of the Patient Activation Measure (PAM): Conceptualizing and Measuring Activation in Patients and Consumers. Health Services Research. 2004;39.

24. Hibbard JH, Mahoney ER, Stockard J, Tusler M. Development and testing of a short form of the patient activation measure. Health Serv Res. 2005;40:1918-30. doi: 10.1111/j.1475-6773.2005.00438.x.

25. Hibbard JH, Mahoney ER, Stock R, Tusler M. Do increases in patient activation result in improved selfmanagement behaviors? Health Serv Res. 2007;42:1443-63. doi: 10.1111/j.1475-6773.2006.00669.x.

26. Hibbard JH, Cunningham PJ. How Engaged Are Consumers in Their Health and Health Care, and Why Does It Matter? Research Brief. 2008;8:1-9. 
27. Hibbard JH, Greene J, Tusler M. Improving the Outcomes of Disease Management by Tailoring Care to the Patient's Level of Activation. Am J Manag Care. 2009;15:353-60.

28. Graffigna G, Barello S, Bonanomi A, Lozza E, Hibbard J. Measuring patient activation in Italy:

Translation, adaptation and validation of the Italian version of the patient activation measure 13 (PAM13I). BMC Med Inform Decis Mak. 2015;15:109. doi: 10.1186/s12911-015-0232-9.

29. Greene J, Hibbard JH, Sacks R, Overton V, Parrotta CD. When patient activation levels change, health outcomes and costs change, too. Health Aff (Millwood). 2015;34:431-7. doi: 10.1377/hlthaff.2014.0452.

30. Smith SG, Pandit A, Rush SR, Wolf MS, Simon C. The association between patient activation and accessing online health information: results from a national survey of US adults. Health Expect. 2014;18:3262-73. doi: 10.1111/hex.12316.

31. Greene J, Hibbard JH. Why Does Patient Activation Matter? An Examination of the Relationships Between Patient Activation and Health-Related Outcomes. J Gen Intern Med 2011;27:520-6. doi: 10.1007/s11606-011-1931-2).

32. Mitchell SE, Gardiner PM, Sadikova E, Martin JM, Jack BW, Hibbard JH, et al. Patient activation and 30-day post-discharge hospital utilization. J Gen Intern Med. 2013;29:349-55. doi: 10.1007/s11606-0132647-2.

33. McCabe PJ, Stuart-Mullen LG, McLeod CJ, T OB, Schmidt MM, Branda ME, et al. Patient activation for self-management is associated with health status in patients with atrial fibrillation. Patient Prefer Adherence. 2018;12:1907-16. doi: 10.2147/PPA.S172970.

34. Yu CH, Parsons J, Mamdani M, Lebovic G, Shah BR, Bhattacharyya O, et al. Designing and evaluating a web-based selfmanagement site for patients with type 2 diabetes - systematic website development and study protocol. BMC Medical Informatics and Decision Making 2012;12:57.

35. Jahangiry L, Shojaeizadeh D, Abbasalizad Farhangi M, Yaseri M, Mohammad K, Najafi M, et al. Interactive web-based lifestyle intervention and metabolic syndrome: findings from the Red Ruby (a randomized controlled trial). Trials. 2015;16:418. doi: 10.1186/s13063-015-0950-4.

36. Whitehead L, Seaton P. The Effectiveness of Self-Management Mobile Phone and Tablet Apps in Long-term Condition Management: A Systematic Review. J Med Internet Res. 2016;18:e97. doi: 10.2196/jmir.4883.

37. Årsand E, Tatara N, Østengen G, Hartvigsen G. Mobile Phone-Based Self-Management Tools for Type 2 Diabetes: The Few Touch Application. Journal of Diabetes Science and Technology. 2010;4.

38. Silveira DV, Marcolino, M.S., Machado, E.L., Ferreira,C.G., Alkmim, M.B.M., Resende, E.S., Carvalho, B.C., Antunes, A.P., Ribeiro, A.L. Development and Evaluation of a Mobile Decision Support System for 
Hypertension Management in the Primary Care Setting in Brazil: Mixed-Methods Field Study on Usability, Feasibility, and Utility. JMIR MHEALTH AND UHEALTH. 2019;7:1. doi: 10.2196/mhealth.9869.

39. Orji R, Moffatt K. Persuasive technology for health and wellness: State-of-the-art and emerging trends. Health Informatics J. 2018;24:66-91. doi: 10.1177/1460458216650979.

40. Chan AW, Tetzlaff JM, Gotzsche PC, Altman DG, Mann H, Berlin JA, et al. SPIRIT 2013 explanation and elaboration: guidance for protocols of clinical trials. BMJ. 2013;346:e7586. doi: 10.1136/bmj.e7586.

41. Eldridge SM, Chan CL, Campbell MJ, Bond CM, Hopewell S, Thabane L, et al. CONSORT 2010 statement: extension to randomised pilot and feasibility trials. BMJ. 2016;355:i5239. doi: 10.1136/bmj.

42. Kendall JM. Designing a research project: randomised controlled trials and their principles. Emerg Med J. 2003;20:164-8.

43. Blatch-Jones AJ, Pek W, Kirkpatrick E, Ashton-Key M. Role of feasibility and pilot studies in randomised controlled trials: a cross-sectional study. BMJ Open. 2018;8:e022233. doi: 10.1136/bmjopen2018-022233.

44. Levati S, Campbell P, Frost R, Dougall N, Wells M, Donaldson C, et al. Optimisation of complex health interventions prior to a randomised controlled trial: a scoping review of strategies used. Pilot Feasibility Stud. 2016;2:17. doi: 10.1186/s40814-016-0058-y.

45. Whitehead AL, Sully BG, Campbell MJ. Pilot and feasibility studies: is there a difference from each other and from a randomised controlled trial? Contemp Clin Trials. 2014;38:130-3. doi:

10.1016/j.cct.2014.04.001.

46. Skivington K, Matthews L, Craig P, Simpson S, Moore L. Developing and evaluating complex interventions: updating Medical Research Council guidance to take account of new methodological and theoretical approaches. The Lancet. 2018;392:S2. doi: 10.1016/S0140-6736(18)32865-4.

47. Craig P, Cooper C, Gunnell D, Haw S, Lawson K, Macintyre S, et al. Using natural experiments to evaluate population health interventions: new Medical Research Council guidance. J Epidemiol Community Health. 2012;66:1182-6. doi: 10.1136/jech-2011-200375.

48. Barjtya S, Sharma A, Rani U. A detailed study of Software Development Life Cycle (SDLC) Models. International Journal Of Engineering And Computer Science 2017;6:22097-100. doi:

10.18535/ijecs/v6i7.32.

49. Chatterjee S, Price A. Healthy living with persuasive technologies: framework, issues, and challenges. J Am Med Inform Assoc. 2009;16:171-8. doi: 10.1197/jamia.M2859.

50. Ali K. A Study of Software Development Life Cycle Process Models. International Journal of Advanced Research in Computer Science. 2017;8:15-23. 
51. Naeem F, Syed Y, Xiang S, Shokraneh F, Munshi T, Yang Y, et al. Development, Testing and Reporting of Mobile Apps for Psycho-social Interventions: Lessons from the Pharmaceuticals. Journal of Medical Diagnostic Methods. 2015;4:4. doi: 10.4172/2168-9784.1000191.

52. Ruggiero KJ, Bunnell BE, Andrews AR, Davidson TM, Hanson RF, Danielson CK, et al. Development and Pilot Evaluation of a Tablet-Based Application to Improve Quality of Care in Child Mental Health Treatment. JMIR Research Protocols. 2015;4. doi: 10.2196/resprot.4416.

53. Bevan N, Carter J, Harker S. ISO 9241-11 Revised: What Have We Learnt About Usability Since 1998 ? In 17th International Conference, $\mathrm{HCl}$ International 2015, Los Angeles, CA, USA, August 2-7, 2015, Proceedings, Part I, edited by Masaaki Kurosu. 2015;Part 1, LNCS 9169 143-51. doi: 10.1007/978-3-31920901-2_13.

54. Clinical Practice Guidelines on the Primary \& Secondary Prevention of Cardiovascular Disease 2017: 1st Edition. Ministry of Health Malaysia. https://www.malaysianheart.org/files/5c4af511bbd3e.pdf.

55. Sohl SJ, Birdee G, Elam R. Complementary Tools to Empower and Sustain Behavior Change: Motivational Interviewing and Mindfulness. Am J Lifestyle Med. 2016;10:429-36. doi: $10.1177 / 1559827615571524$.

56. Wolever RQ, Simmons LA, Sforzo GA, Dill D, Kaye M, Bechard EM, et al. A Systematic Review of the Literature on Health and Wellness Coaching: Defining a Key Behavioral intervention in Healthcare. Glob Adv Health Med. 2013;2:38-57. doi: 10.7453/gahmj.2013.042.

57. Boehmer KR, Barakat S, Ahn S, Prokop LJ, Erwin PJ, Murad MH. Health coaching interventions for persons with chronic conditions: a systematic review and meta-analysis protocol. Syst Rev. 2016;5:146. doi: 10.1186/s13643-016-0316-3.

58. Simmons LA, Wolever RQ. Integrative Health Coaching and Motivational interviewing: Synergistic Approaches to Behavior Change in Healthcare. Glob Adv Health Med. 2013;2:28-35. doi: 10.7453/gahmj.2013.037.

59. Graham ID, Logan J, Harrison MB, Straus SE, Tetroe J, Caswell W, et al. Lost in Knowledge Translation: Time for a Map? The Journal of Continuing Education in the Health Professions. 2006;26. doi: 10.1002/chp.4710.1002/chp.

60. Shamsuddin N, Koon PB, Syed Zakaria SZ, Noor MS, Jamal R. Reliability and Validity of Malay Language Version of International Physical Activity Questionnaire (IPAQ-M) among the Malaysian Cohort Participants. International Journal of Public Health Research. 2015;5:643-53.

61. Subramaniam K, Low WY, Chinna K, Chin KF, Krishnaswamy S. Psychometric Properties of the Malay Version of the Dutch Eating Behaviour Questionnaire (DEBQ) in a Sample of Malaysian Adults Attending a Health Care Facility. Malays J Med Sci. 2017;24:64-73. doi: 10.21315/mjms2017.24.4.8. 
62. Abdul-Razak S, Ramli AS, Badlishah-Sham SF, Haniff J. Validity and reliability of the patient assessment on chronic illness care (PACIC) questionnaire: the Malay version. BMC Fam Pract. 2018;19:119. doi: 10.1186/s12875-018-0807-5.

63. Abd Aziz A, Nordin NIF, Mohd Noor N, Bachok N, Isa NIS. Psychometric properties of the 'Skala Kepuasan Interaksi Perubatan-11' to measure patient satisfaction with physician-patient interaction in Malaysia. Fam Pract. 2014;31:236-44. doi: 10.1093/fampra/cmt062.

64. Frijlinga BD, Lobob CM, Keusa IM, Jenksa KM, Akkermansa RP, Hulschera MEJL, et al. Perceptions of cardiovascular risk among patients with hypertension or diabetes. Patient Education and Counseling 2004;52:47-53. doi: 10.1016/S0738-3991(02)00248-3.

65. D'Agostino RB, Sr., Vasan RS, Pencina MJ, Wolf PA, Cobain M, Massaro JM, et al. General cardiovascular risk profile for use in primary care: the Framingham Heart Study. Circulation. 2008;117:743-53. doi: 10.1161/CIRCULATIONAHA.107.699579.

66. Martell-Claros N, Aranda P, Gonzalez-Albarran O, Dalfo-Baque A, Dominguez-Sardina M, de la Cruz JJ, et al. Perception of health and understanding of cardiovascular risk among patients with recently diagnosed diabetes and/or metabolic syndrome. Eur J Prev Cardiol. 2013;20:21-8. doi: $10.1177 / 1741826711423114$.

67. Malaysian Guideline for Good Clinical Practice: 4th Edition. National Pharmaceutical Regulatory Agency (NPRA), Ministry of Health Malaysia.

68. Craig CL, Marshall AL, Sjostrom M, Bauman AE, Booth ML, Ainsworth BE, et al. International physical activity questionnaire: 12-country reliability and validity. Med Sci Sports Exerc. 2003;35:1381-95. doi: 10.1249/01.MSS.0000078924.61453.FB.

69. The International physical activity questionnaire. Guidelines for data processing and analysis of the International Physical Activity Questionnaire (IPAQ). Revised 2005. http://www.ipaq.ki.se/scoring.pdf

70. van Strien T, Frijters JER, Bergers GPA, Defares PB. The Dutch Eating Behavior Questionnaire (DEBQ) for Assessment of Restrained, Emotional, and External Eating Behavior. Int J Eat Disorder. 1986;5:295315. doi: doi.org/10.1002/1098-108x(198602)5:2<295::aideat2260050209>3.0.co;2-t.

71. Glasgow RE, Wagner EH, Schaefer J, Mahoney LD, Reid RJ, Greene SM. Development and Validation of the Patient Assessment of Chronic Illness Care (PACIC). Med Care. 2005;43:436-44.

72. Meakin R. The 'Medical Interview Satisfaction Scale' (MISS-21) adapted for British general practice. Family Practice. 2002;19:257-63. doi: 10.1093/fampra/19.3.257.

73. Chia YC, Gray SY, Ching SM, Lim HM, Chinna K. Validation of the Framingham general cardiovascular risk score in a multiethnic Asian population: a retrospective cohort study. BMJ Open. 2015;5:e007324. doi: 10.1136/bmjopen-2014-007324. 
74. Dupont WD, Plummer WD. Power and sample size calculations. Controlled Clinical Trials. 1990;11:116-28. doi: 10.1016/0197-2456(90)90005-m.

75. Solomon M, Wagner SL, Goes J. Effects of a Web-Based Intervention for Adults With Chronic Conditions on Patient Activation: Online Randomized Controlled Trial. Journal of Medical Internet Research. 2012;14. doi: 10.2196/jmir.1924.

76. Bland JM, Altman DG. Measuring agreement in method comparison studies. Stat Methods Med Res. 1999;8:135-60. doi: 10.1177/096228029900800204.

77. Bodenheimer T, Wagner EH, Grumbach K. Improving primary care for patients with chronic illness. JAMA. 2002;288:1775-9.

78. Daily mobile app usage in Asia Pacific 2016, by country. Statista Research Department Retrieved on 19th October 2018. https://www.statista.com/statistics/293661/daily-mobile-app-usage-in-apaccountries/.

79. Li I, Dey A, Forlizzi J. A Stage-based Model of Personal Informatics Systems. In Proceedings of the SIGCHI Conference on Human Factors in Computing Systems (CHI '10) 2010, Atlanta, Georgia, USA.55766 .

80. MacLeod H, Tang A, Carpendale S. Personal informatics in chronic illness management. In Proceedings of Graphics Interface 2013. Canadian Information Processing Society.149-56.

81. Morris ME, Kathawala Q, Leen TK, Gorenstein EE, Guilak F, Labhard M, et al. Mobile therapy: case study evaluations of a cell phone application for emotional self-awareness. J Med Internet Res. 2010;12:e10. doi: 10.2196/jmir.1371.

82. Morton K, Beauchamp M, Prothero A, Joyce L, Saunders L, Spencer-Bowdage S, et al. The effectiveness of motivational interviewing for health behaviour change in primary care settings: a systematic review. Health Psychol Rev. 2015;9:205-23. doi: 10.1080/17437199.2014.882006.

83. Ramli AS, Lakshmanan S, Haniff J, Selvarajah S, Tong SF, Bujang MA, et al. Study protocol of EMPOWER Participatory Action Research (EMPOWER-PAR): a pragmatic cluster randomised controlled trial of multifaceted chronic disease management strategies to improve diabetes and hypertension outcomes in primary care. BMC Family Practice 2014;15:1-16.

84. Jung $\mathrm{H}$, Lee JE. The impact of community-based eHealth self-management intervention among elderly living alone with hypertension. J Telemed Telecare. 2017;23:167-73. doi: $10.1177 / 1357633 \times 15621467$.

85. Varnfield M, Karunanithi M, Lee CK, Honeyman E, Arnold D, Ding H, et al. Smartphone-based home care model improved use of cardiac rehabilitation in postmyocardial infarction patients: results from a randomised controlled trial. Heart. 2014;100:1770-9. doi: 10.1136/heartjnl-2014-305783. 
86. Glasgow RE, Kurz D, King D, Dickman JM, Faber AJ, Halterman E, et al. Twelve-month outcomes of an Internet-based diabetes self-management support program. Patient Educ Couns. 2012;87:81-92. doi: 10.1016/j.pec.2011.07.024.

87. Quinn CC, Shardell MD, Terrin ML, Barr EA, Ballew SH, Gruber-Baldini AL. Cluster-Randomized Trial of a Mobile Phone Personalized Behavioral Intervention for Blood Glucose Control. Diabetes Care. 2011;34:1934-42.

88. Hägglun E, Lyngå P, Frie F, Ullman B, Persson H, Melin M, et al. Patient-centred home-based management of heart failure: Findings from a randomised clinical trial evaluating a tablet computer for self-care, quality of life and effects on knowledge. Scandinavian Cardiovascular Journal. 2015;49:1-18. doi: 10.3109/14017431.2015.1035319.

89. Waki K, Fujita H, Uchimura Y, Omae K, Aramaki E, Kato S, et al. DialBetics: A Novel Smartphone-based Self-management Support System for Type 2 Diabetes Patients. J Diabetes Sci Technol. 2014;8:209-15. doi: $10.1177 / 1932296814526495$.

90. Liu WT, Huang CD, Wang CH, Lee KY, Lin SM, Kuo HP. A mobile telephone-based interactive self-care system improves asthma control. Eur Respir J. 2011;37:310-7. doi: 10.1183/09031936.00000810.

\section{Table 1}

Table 1: The EMPOWER-SUSTAIN Self-Management e-Health Intervention 


\begin{tabular}{|c|c|c|}
\hline $\begin{array}{l}\text { Chronic } \\
\text { Care Model } \\
\text { Elements }\end{array}$ & Crucial Components & Intervention \\
\hline $\begin{array}{l}\text { Decision } \\
\text { Support }\end{array}$ & $\begin{array}{l}\text { Decision support for } \\
\text { physicians to translate } \\
\text { clinical practice } \\
\text { guidelines (CPG) } \\
\text { recommendations into } \\
\text { day to day clinical } \\
\text { practice [75, 81-82] }\end{array}$ & $\begin{array}{l}\text { EMPOWER-SUSTAIN Training Workshop for } \\
\text { PCP to use web-based desktop and mobile } \\
\text { applications; and to reinforce the use of relevant } \\
\text { CPG for management and prescribing }\end{array}$ \\
\hline $\begin{array}{l}\text { Self- } \\
\text { Management } \\
\text { Support }\end{array}$ & $\begin{array}{l}\text { Self-Management } \\
\text { support to facilitate } \\
\text { patient activation and } \\
\text { behaviour change [84- } \\
\text { 87] }\end{array}$ & $\begin{array}{l}\text { Primary Care Physicians (PCP) to support and } \\
\text { empower patients with knowledge, skills and } \\
\text { confidence in utilising the EMPOWER-SUSTAIN } \\
\text { mobile application and the EMPOWER-SUSTAIN } \\
\text { Global CV Risks Self-Management Booklet }{ }^{\odot} \\
\text { using the persuasive technology approach }\end{array}$ \\
\hline $\begin{array}{l}\text { Delivery } \\
\text { System } \\
\text { Design }\end{array}$ & $\begin{array}{l}\text { Delivery system design } \\
\text { through the } \\
\text { EMPOWER-SUSTAIN } \\
\text { Clinic to improve } \\
\text { continuity of care and } \\
\text { patient-physician } \\
\text { relationship [88-90] }\end{array}$ & $\begin{array}{l}\text { The EMPOWER-SUSTAIN Self-Management e- } \\
\text { Health Intervention will be delivered by PCP at } \\
\text { baseline, 3-month and 6-month follow-up in the } \\
\text { EMPOWER-SUSTAIN Clinic to ensure continuity } \\
\text { of care and sustainability }\end{array}$ \\
\hline
\end{tabular}

\section{Additional File Information}

Additional File 1: The EMPOWER-SUSTAIN SPIRIT checklist

Additional File 2: The EMPOWER-SUSTAIN mobile app mock prototype

Additional File 3: The EMPOWER-SUSTAIN patient consent form

Additional File 4: The EMPOWER-SUSTAIN physician consent form

\section{Figures}




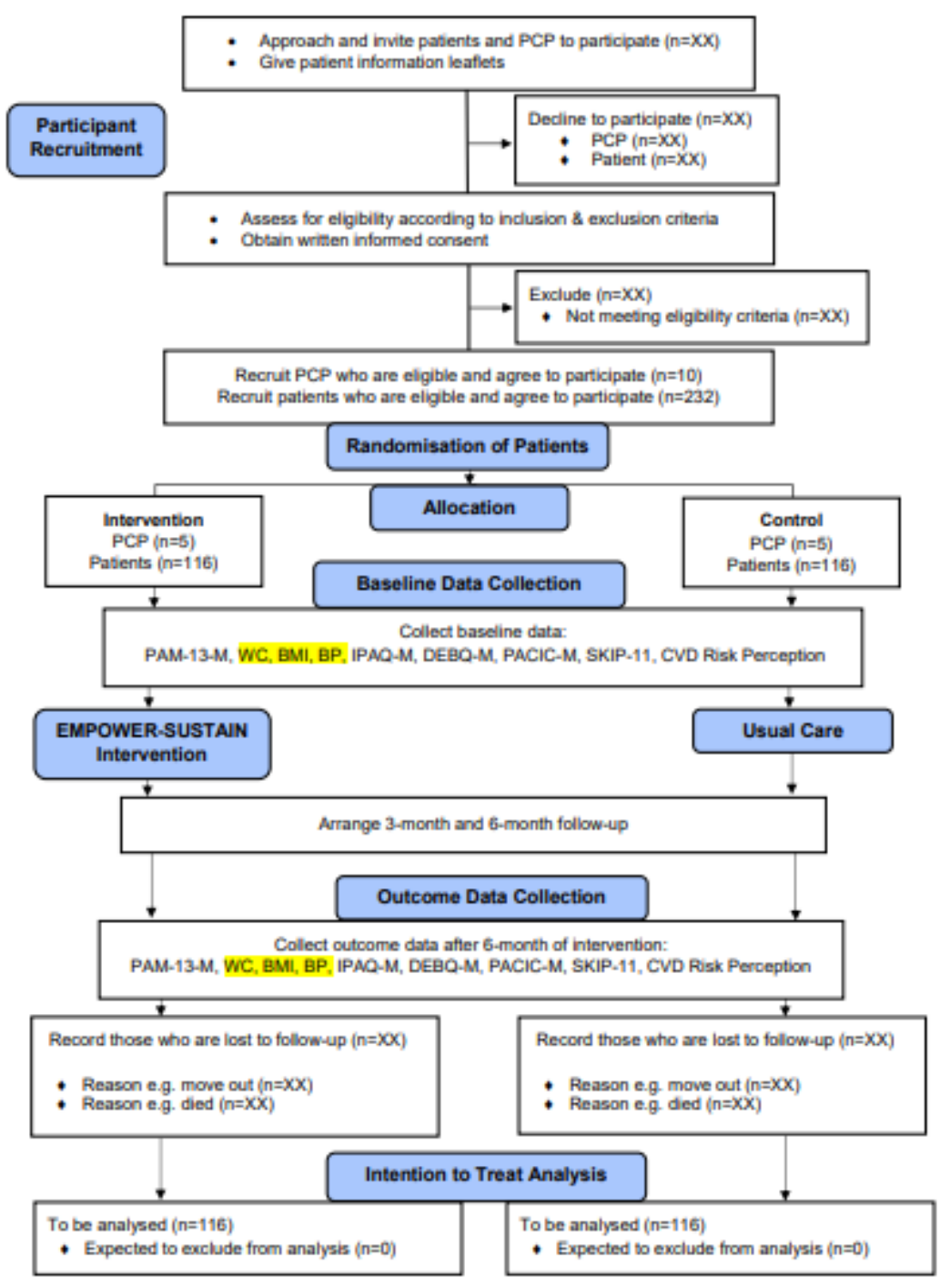

\section{Figure 1}

The EMPOWER-SUSTAIN CONSORT flow diagram 


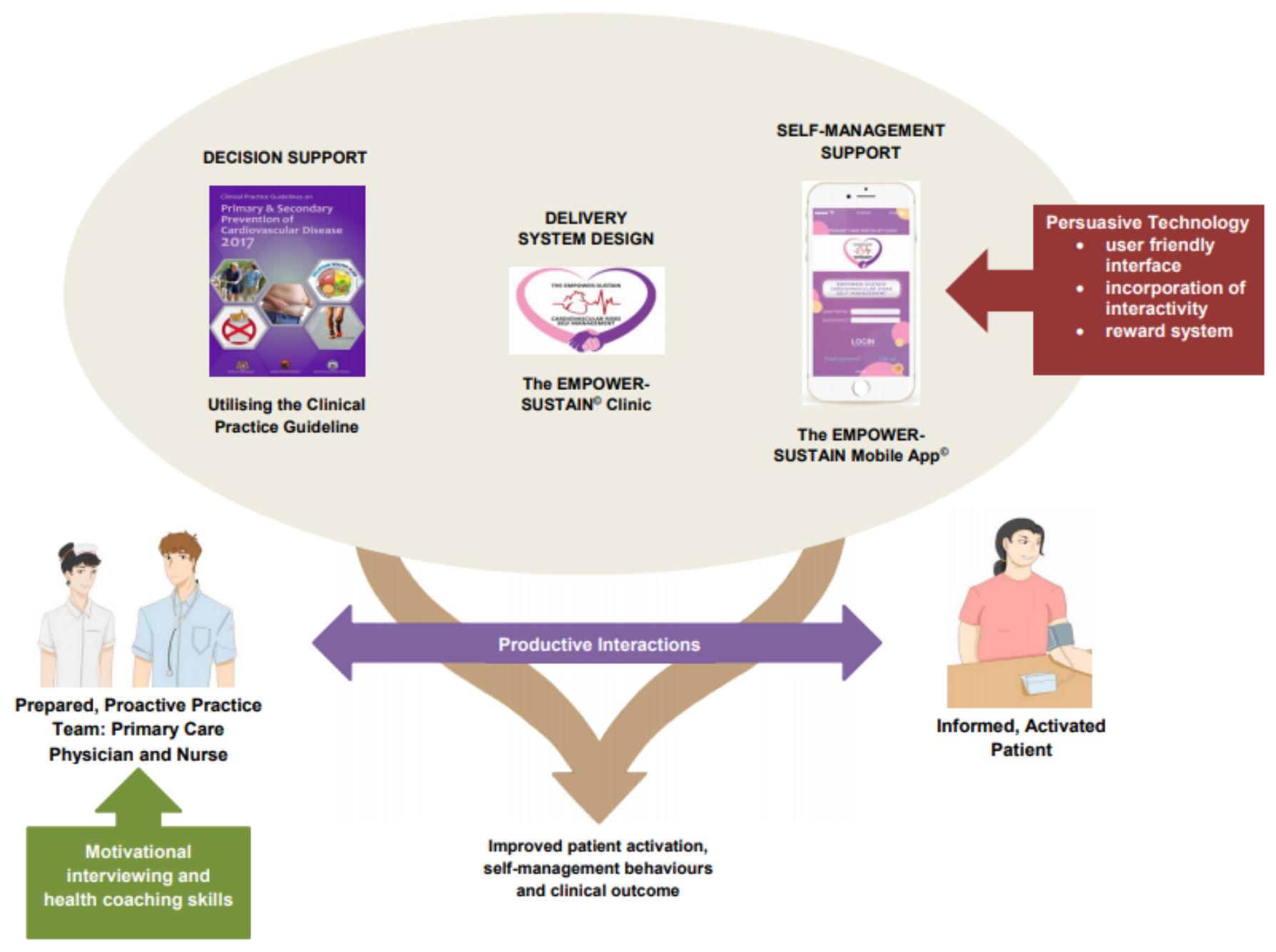

\section{Figure 2}

The conceptual framework for the EMPOWER-SUSTAIN Self-Management e-Health Intervention 


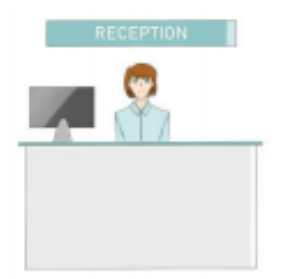

Receptionist registers patient using the EMPOWER-SUSTAIN ${ }^{\circ}$ desktop application

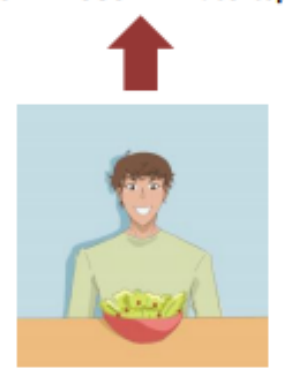

Self-Management: Patient eats healthy diet
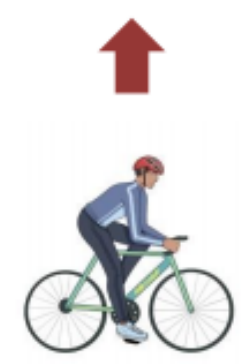

Self-Management:

Patient exercises at home or in the neighbourhood

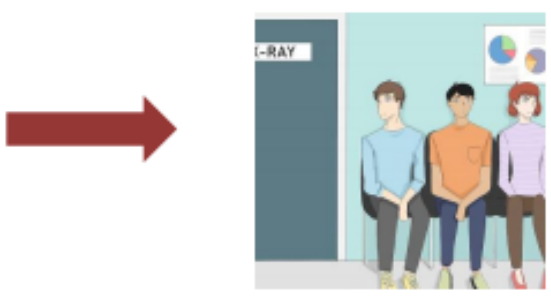

Patient waits in the waiting room

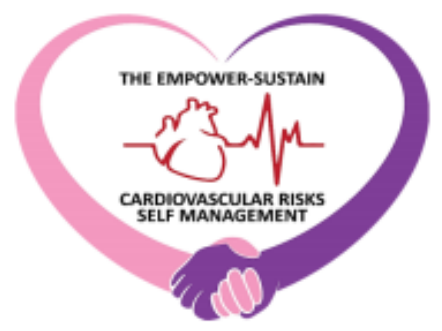

The EMPOWER-SUSTAIN ${ }^{\circledR}$ e-Health Intervention

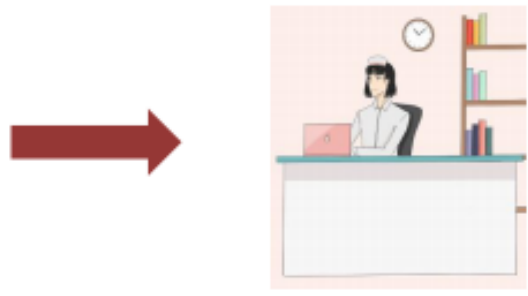

Nurse enters check-up data using the EMPOWER-SUSTAIN ${ }^{\circ}$ desktop application
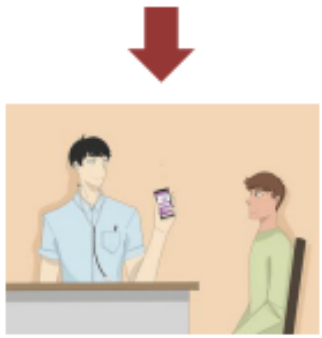

Doctor consults patient using the EMPOWER-SUSTAIN ${ }^{\circ}$ desktop and mobile applications

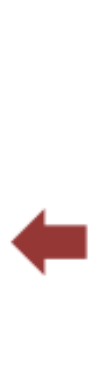
Patient checks blood pressure

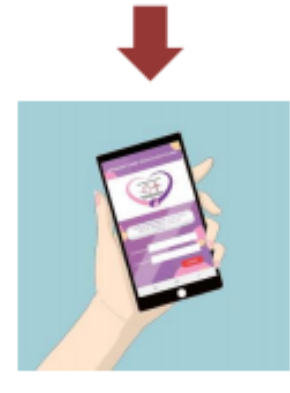

Self-Management: Patient uses the EMPOWERSUSTAIN $^{\circ}$ mobile app at home
Self-Management:

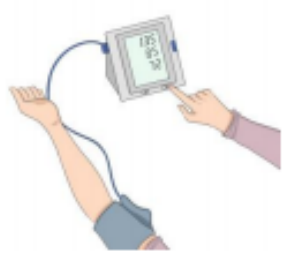

Self-Management: Patient checks blood sugar at home
Patient checks blood
at home

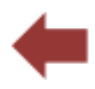

\section{Figure 3}

Delivery of the EMPOWER-SUSTAIN Self-Management e-Health Intervention 


\begin{tabular}{|c|c|c|c|c|c|c|}
\hline & \multicolumn{6}{|c|}{ STUDY PERIOO } \\
\hline & \multirow{3}{*}{$\begin{array}{l}\text { Enrolmont } \\
-3 \text { manth }\end{array}$} & \multirow{3}{*}{$\begin{array}{c}\text { Allocation } \\
0\end{array}$} & \multicolumn{3}{|c|}{ Post-allocation } & \multirow{3}{*}{$\begin{array}{c}\begin{array}{c}\text { Post- } \\
\text { intervention }\end{array} \\
\begin{array}{c}\text { Outcome } \\
\text { data } \\
\text { collection }\end{array} \\
6-8 \text { month }\end{array}$} \\
\hline & & & \multirow{2}{*}{$\begin{array}{c}\begin{array}{c}\text { Baseine } \\
\text { data } \\
\text { collection }\end{array} \\
1-2 \text { month }\end{array}$} & \multicolumn{2}{|c|}{$\begin{array}{l}\text { The EMPOWER } \\
\text { SUSTAIN E-Health } \\
\text { Insorvention }\end{array}$} & \\
\hline TIMEPOINT & & & & $\begin{array}{l}\text { 3-month } \\
\text { follow-wp }\end{array}$ & $\begin{array}{l}\text { 6-month } \\
\text { follow up }\end{array}$ & \\
\hline \multicolumn{7}{|l|}{ ENROLNENT: } \\
\hline \multirow{3}{*}{$\begin{array}{l}\text { Eligibility screen } \\
\text { Informed consent } \\
\text { The EMPOWER. } \\
\text { SUSTAN } \\
\text { Workshop for the } \\
\text { Primary Care } \\
\text { Physicians }\end{array}$} & $\mathrm{x}$ & & & & & \\
\hline & $\mathrm{x}$ & & & & & \\
\hline & $\mathrm{x}$ & & & & & \\
\hline $\begin{array}{r}\text { Randomization } \\
\text { and Allocation of } \\
\text { Patients }\end{array}$ & & $x$ & & & & \\
\hline \\
\hline \multirow{2}{*}{$\begin{array}{r}\text { The EMPOWER. } \\
\text { SUSTAW e-Hoaim } \\
\text { intervention } \\
\text { Control }\end{array}$} & & & $\mathrm{x}$ & & & $\mathrm{x}$ \\
\hline & & & $\mathrm{x}$ & & & $\mathrm{x}$ \\
\hline \multicolumn{7}{|l|}{ AsSESSMENTS: } \\
\hline $\begin{array}{r}\text { Sociodemographic } \\
\text { and } \\
\text { anthropometric } \\
\text { data }\end{array}$ & $\mathrm{x}$ & & & & & \\
\hline $\begin{array}{l}\text { Primary Outceme: } \\
\text { PAM-13-1 }\end{array}$ & & & $\mathrm{x}$ & & & $\mathrm{x}$ \\
\hline $\begin{array}{r}\text { Secondary } \\
\text { Outcames: WC, } \\
\text { BMU, BP, IPAOA-M, } \\
\text { DEBQ-K, PACIC-M, } \\
\text { SKOP-11, CVD Risk } \\
\text { Pereeption }\end{array}$ & & & $\mathrm{x}$ & & & $\mathrm{x}$ \\
\hline
\end{tabular}

Abbrewations: PAM-13-M, Pabent Activation Measure shant form Malay version; WC, Waist

Circumference; EME. Body Maras index: BP, Blood Presaure, IPAQ-M, ntemarional Physical Activity

Ouestionnaire Malay version, DEBQ-M. Duich Eafing Behaviour Ouestionnaire - Maiay version, PACIC.

M, Patienss Asseasment af Chronic Ilness Care - Malay version; SKIP-11, Skala Kepuasan interaksi

Pentbatan 11; CVD, Cardiowascular disease.

\section{Figure 4}

The EMPOWER-SUSTAIN SPIRIT schedule of enrolment, intervention and assessment

\section{Supplementary Files}

This is a list of supplementary files associated with this preprint. Click to download.

- AdditionalFile2EMPOWERSUSTAINMockPrototype.pdf

- AdditionalFile2EMPOWERSUSTAINMockPrototype.pdf

- AdditionalFile4EMPOWERSUSTAINPCPNurseConsentForm.doc

- AdditionalFile3EMPOWERSUSTAINPatientConsentForm.doc

- AdditionalFile1EMPOWERSUSTAINSPIRITChecklist.doc 